\title{
Anti-cancer Activity of Two Novel Heterocyclic Compounds through Modulation of VEGFR and miRNA-122 in Mice Bearing Ehrlich Ascites Carcinoma
}

\author{
Reem Hazem ${ }^{1}$, Anhar Ali ${ }^{2}$, Eman mahana ${ }^{1}$, Noha Mesbah $^{1}$, Dina Abouelmouti ${ }^{1}$, and \\ Mohamed Elgawish ${ }^{1}$ \\ ${ }^{1}$ Suez Canal University Faculty of Pharmacy \\ ${ }^{2}$ Sinai University - El Arish Campus
}

April 28, 2020

\begin{abstract}
Background and Purpose: Metastasis in breast cancer is a leading cause of mortality among women in many countries. This study investigated the anti-cancer role of benzoimidazoquinazoline and benzimidazotriazin; two novel compounds that were designed, synthesized, structurally elucidated, and biologically evaluated as potent anti-angiogenic agents that act through inhibition of vascular endothelial growth factor receptor-2 (VEGFR2). A model of breast cancer was induced by inoculation of Ehrlich Ascites Carcinoma (EAC) cells. Experimental Approach: Seventy swiss albino mice were randomly divided into 7 groups, 10 animals each: (1) normal, (2) control EAC group, (3) cisplatin treated group, (4\&5) benzoimidazoquinazoline treated $(5 \mathrm{mg} / \mathrm{kg}$ and $10 \mathrm{mg} / \mathrm{kg}),(6 \& 7)$ benzimidazotriazin treated $(5 \mathrm{mg} / \mathrm{kg}$ and $10 \mathrm{mg} / \mathrm{kg})$. The expression of miRNA-122 was assessed in the tumor tissue by quantitative PCR, and the VEGF level was determined in serum by ELISA. VEGFR2 and cluster of differentiation (CD)34 were assessed by immunohistochemistry. Serum levels of ALT, AST, creatinine, and urea were measured. Key Results: Treatment with benzoimidazoquinazoline and benzimidazotriazin caused a decrease in tumor weight and a significant decrease in the serum levels of VEGF and the expression of VEGFR2 and CD34 in the tumor tissue. MiRNA-122 was significantly upregulated especially in the group treated by benzimidazotriazin (10mg/kg). Interestingly, the new compounds had less renal toxicity compared to cisplatin. Conclusion and Implication: The designed small molecules are promising anti-cancer candidates that act through inhibition of angiogenesis and can provide a new strategy for the advancement of chemotherapy through modulation of miRNA.
\end{abstract}

\section{ABSTRACT}

Background and Purpose: Metastasis in breast cancer is a leading cause of mortality among women in many countries. This study investigated the anti-cancer role of benzoimidazoquinazoline and benzimidazotriazin; two novel compounds that were designed, synthesized, structurally elucidated, and biologically evaluated as potent anti-angiogenic agents that act through inhibition of vascular endothelial growth factor receptor-2 (VEGFR2). A model of breast cancer was induced by inoculation of Ehrlich Ascites Carcinoma (EAC) cells.

Experimental Approach: Seventy swiss albino mice were randomly divided into 7 groups, 10 animals each: (1) normal, (2) control EAC group, (3) cisplatin treated group, (4\&5) benzoimidazoquinazoline treated $(5 \mathrm{mg} / \mathrm{kg}$ and $10 \mathrm{mg} / \mathrm{kg}),(6 \& 7)$ benzimidazotriazin treated $(5 \mathrm{mg} / \mathrm{kg}$ and $10 \mathrm{mg} / \mathrm{kg})$. The expression of miRNA-122 was assessed in the tumor tissue by quantitative PCR, and the VEGF level was determined in serum by ELISA. VEGFR2 and cluster of differentiation (CD)34 were assessed by immunohistochemistry. Serum levels of ALT, AST, creatinine, and urea were measured. 
Key Results: Treatment with benzoimidazoquinazoline and benzimidazotriazin caused a decrease in tumor weight and a significant decrease in the serum levels of VEGF and the expression of VEGFR2 and CD34 in the tumor tissue. MiRNA-122 was significantly upregulated especially in the group treated by benzimidazotriazin $(10 \mathrm{mg} / \mathrm{kg})$. Interestingly, the new compounds had less renal toxicity compared to cisplatin.

Conclusion and Implication: The designed small molecules are promising anti-cancer candidates that act through inhibition of angiogenesis and can provide a new strategy for the advancement of chemotherapy through modulation of miRNA.

Key words : Breast cancer; Angiogenesis; VEGF; VEGFR-2; miRNA-122; Benzoimidazoquinazoline; Benzimidazotriazin.

\section{INTRODUCTION}

Breast cancer is the most widespread cancer among women around the world, affecting more than 2 million new cases and resulting in 600,000 deaths in 2018 (Bray et al., 2018 ). Despite the increased number of patients treated with surgery, radiotherapy or chemotherapeutics, $5-11 \%$ of patients exist with metastatic disease (Tao et al., 2017 ), and a large number of early breast cancer patients have a micro-metastatic disease resistant to systemic treatment. These patients may eventually relapse. New treatment strategies of breast cancer are required (Colozza et al., 2007).

Angiogenesis and lymphangiogenesis stimulate the formation of new blood and lymph vessels from preexisting vasculature. They occur through the proliferation, migration, and maturation of nearby blood or lymph vessel endothelial cells (Lala et al., 2018). Angiogenesis is a basic biological process that is essential for development, reproduction, and wound repair. Angiogenesis is a key feature and distinctive marker of cancer (Otrock et al., 2007 ). Since tumors cannot grow to 1-2 mm without an adequate blood supply, angiogenesis is fundamental for uncontrolled growth of tumors to supply of adequate oxygen and nutrients (Lou et al., 2017 ) Similarly, lymphangiogenesis is involved in many cancer types, including breast cancer. The primary sites of metastasis in breast cancer are often the regional lymph nodes, where lymphangiogenesis can facilitate the migration of tumor cells to these sites (Ran et al., 2010) In the last two decades, vascular endothelial growth factors (VEGFs) and their receptors (VEGFRs) have been identified as the key drivers of lymphangiogenesis and angiogenesis in the vascular systems (Shibuya et al., 2011 ) The VEGF signaling pathway is a crucial regulator in many tissues and plays a vital role in the pathogenesis of cancer, cardiovascular and intraocular neovascular diseases.

VEGFRs play a vital role in vasculogenesis and angiogenesis in embryonic development, wound repair, menstruation, and pregnancy. VEGFR-2, a member of the VEGFR family, helps in the generation of new blood vessels from existing tumor mass. Disruption of VEGFR-2 signaling has resulted in inhibition of angiogenesis and prevention of oxygen, nutrients from tumor cells, and decreasing clearance of catabolic products from tumor cells (Ferrara et al., 2010). Inhibition of VEGF/VEGFR signaling cascade has emerged as an attractive therapeutic tactic for inhibition of tumor angiogenesis and tumor proliferation (Ferrara et al., 2010, Olsson et al, 2006 ) Additionally, the cluster of differentiation (CD) 34 is a specific biomarker of cellular vascular endothelium. CD34 is especially sensitive to tumor angiogenesis, as it can clearly identify the condition of neovascularization during tumor growth (Zorgetto, 2013). In this manner, CD34 and VEGF are two significant markers of tumor angiogenesis and the relationship between the expression of CD34 and VEGF and the pathological attributes of patients with malignancy have been recently reported (Chen et al., 2015 ) Many molecules have been implicated in angiogenesis regulation. Among them, VEGF is the most pivotal angiogenic factor and their expression is regulated by many factors such as hypoxia-inducible factors, chemokines, and microRNAs (Olsson et al, 2006, Zhong et al., 2006)

MicroRNAs (miRNAs) are small non-coding RNA molecules 22 nucleotides in length (Hu et al., 2017 ) MiRNAs play a major role in the regulation of the expression of 30-60\% of human genes and are considered as important modulators of cell differentiation, proliferation, cell-cycle progression, epithelial-mesenchymal transition, angiogenesis, stem cell renewal, apoptosis and cell migration, invasion, and metastasis (Hamam et al., 2017 ) Imbalance in miRNA expression is associated with several diseases such as cancers, where 
they can act as promoters or suppressors of tumorigenesis (Hamam et al., 2017, Song et al., 2013 ). Many miRNAs have been reported to be downregulated in malignant tumors including lung, breast, ovarian, bladder and colon. Dysregulation in the miRNA machinery is a plausible cause for the beginning and evolution of human cancers (Svoronos et al., 2016, Kian et al., 2018 ). MiRNAs can be biological markers for diagnosis, follow-up, and prognosis prediction of cancer patients. miRNA-122 is downregulated in many types of cancer, including liver, breast and renal cancer (Pan et al., 2016, Ahsani et al., 2017, Maierthaler et al., 2017 ) In breast cancer, miRNA-122 was suggested to act as a tumor suppressor and to inhibit the tumorigenesis through targeting insulin-like growth factor 1 receptor (IGF1R) and regulating PI3K/Akt/mTOR/p70S6K pathway (Wang et al., 2012 )

Since VEGF/VEGFR signaling pathway and miRNAs have been utilized as a valuable target for cancer management, developing novel molecules with dual activity is important. Inhibition of VEGFR occurs through two mechanisms. The first mechanism is to block ligand binding to the extracellular domain with monoclonal antibodies (e.g. bevacizumab). The other mechanism relies on using small-molecule receptor tyrosine kinase (TK) inhibitors that function at intracellular domain. Gefitinib, Sorafenib, pazopanib, and Sunitinib are 4-anilinoquinazoline derivatives which competitively bind to the adenosine triphosphate (ATP) binding pocket of intracellular VEGFR domain (Fig 1), thereby inhibiting downstream signaling essential for tumor survival and proliferation (Musumeci et al., 2012, Fan-Wei et al., 2017 ) Despite high response rate of patients to these agents, drug resistance, which occurs because of a secondary mutation, limits the therapeutic benefits of these drugs. Therefore, development of second-generation VEGFR tyrosine kinase inhibitors through the discovery of new scaffold can be beneficial for gefitinib-resisting patients.

Modulations to the central core pyridine ring becomes a feature of the medicinal chemistry strategy to look for potency, structure-activity relationship (SAR), and reducing log p of TK inhibitors. A range of heteroatoms have been reported to provide alternatives to the pyridine and/or pyrimidine ring of Sorafenib and Pazopanib. Amongst these rings, quinazoline and naphtamide derivatives, Furo[2,3-d] pyrimidines, pyridinyltriazines, and pyrimidinylindazoles have been reported as VGEFR-2 inhibitor. ${ }^{24}$ In the current study, diverse structure modifications in hit compounds have been studied to improve its potency against VEGFR. On another hand, unusual regulation of microRNAs has been connected to different human diseases, in particular cancer. Small molecule mediation of microRNA misregulation thus can give new therapeutic approaches to manage such disease (Holmes et al., 2007). In this study, the effect of two novel compounds, benzoimidazoquinazoline and benzimidazotriazin in treatment of breast cancer induced in mice was studied. Their effect on the expression of miRNA-122, CD34, VEGFR, and VEGF in the tumor tissues was assessed.

\section{EXPERIMENTAL SECTION}

2. Chemicals and reagents:

The synthesized compounds used in treatment (benzoimidazoquinazoline and benzimidazotriazine) were provided by the medicinal chemistry department and the complete physicochemical data are provided in supporting information. Cisplatin was purchased from "EIMC united pharmaceuticals, Badr City, Cairo, Egypt". Disodium EDTA was bought from Alpha Chem (USA). Phosphate buffered saline (PBS) was purchased from BioWhittaker R Lonza (Switzerland). Monoclonal mouse antibodies for CD34 VEGFR-2 were purchased from Santa Cruz Biotechnology (Santa Cruz, CA, USA).

\section{Synthesis of designed compounds}

Benzoimidazoquinazoline $(\mathrm{C} 1)$ and benzimidazotriazine $(\mathrm{C} 2)$ molecules were synthesized for initial testing as a promising anticancer lead molecule modulating the activity of VEGFR, CD34, and microRNA-122; full synthesis and characterization details are given in the Supporting Information . o-Phenylenediamine was refluxed with an equimolar concentration of anthranilic acid producing 2-(1H-benzo[d]imidazol-2-yl) aniline in high yield. The 2-(1H-benzo[d]imidazol-2-yl) aniline then was condensed with carbon disulfide in $\mathrm{KOH}$ producing a cyclized benzo[4,5]imidazo[1,2-c]quinazoline-6-thiol. The free thiol was reacted with chloroacetylchloride under basic condition to release S-(benzo[4,5]imidazo[1,2-c]quinazolin-6-yl) 2-chloroethanethioate in reasonable yield. Finally, chloroethanethioate derivative was condensed in basic media with dichloroani- 
line giving the target compound (C1). Similarly, o -phenylenediamine was condensed in acidic media with 2-mercaptoacetic acid releasing (1H-benzo[d]imidazol-2-yl)methanethiol in good yield, which then refluxed for $20 \mathrm{hr}$. with an excess concentration of hydrazine hydrate in ethanol producing 2-(hydrazinylmethyl)$1 \mathrm{H}$-benzo[d]imidazole. The hydrazine derivative was cyclized producing imidazo[1,2-d][1,2,4]triazine after condensation with trimethoxymethane. Lastly, the cyclized triazine was condensed with salicylaldehyde producing compound C2 in good yield. The complete reaction steps, condition, and yields are found in Scheme-1

\section{Tumor cells:}

Human breast cancer cell line MCF-7 and Ehrlich Ascites Carcinoma (EAC) cells were purchased from Tumor Biology Department, National Cancer Institute, Cairo University. The cells were cultured in Dulbecco's modified Eagle's medium (DMEM) supplemented with $10 \%$ heat-inactivated fetal bovine serum, $1 \%$ Lglutamine, HEPES buffer, and $50 \mu \mathrm{g} / \mathrm{ml}$ gentamycin. All cells were maintained at $37^{\circ} \mathrm{C}$ in a fully humidified air atmosphere containing $5 \% \mathrm{CO}_{2}$ and were sub-cultured two times a week.

EAC is a murine spontaneous breast cancer that served as the original tumor from which an ascites variant was obtained. On intraperitoneal inoculation, an ascitic fluid rich in tumor cells was produced. The tumor cell line was maintained in our laboratory by serial intraperitoneal passages into Swiss female albino mice at 7-10 days interval.

\section{Experimental Animals:}

Seventy swiss albino mice weighing 25-30g were obtained from the Egyptian Organization for Biological Products and Vaccines (Vacsera, Egypt). Animals were housed in plastic cages with mesh floor and hardwood bedding. They were kept under controlled laboratory conditions with normal light/dark cycle at $25-30^{\circ} \mathrm{C}$. Food and water were provided ad libitum during the study period. Mice were left to acclimatize for 1 week before the experiments. All animal procedures and experimental protocols were carried out in accordance with the Guide for the Care and Use of Laboratory animals. The study was approved by the ethical committee of Faculty of Pharmacy, Suez Canal University (201704AM1)

\section{In vitro study:}

Anticancer activity of imidazothiazole and pyridazobenzimidazole was tested in the MCF-7 cell line by using HTScan $\cap$ VEGF Receptor 2 Kinase Assay Kit. The kit provides a means of performing kinase activity assays with recombinant human VEGFR-2 kinase. It includes active VEGFR-2 kinase (supplied as a GST fusion protein), a biotinylated peptide substrate and a phospho-tyrosine antibody for detection of the phosphorylated form of the substrate peptide. Products Included were Phospho-Tyrosine Mouse mAb (P-Tyr-100), HTScanß Tyrosine Kinase Buffer (4X), DTT (1000x, 1.25 M), ATP (10 mM), Gastrin Precursor (Tyr87) Biotinylated Peptide, VEGF Receptor 2 Kinase (recombinant, human).

\section{Preparation of Ehrlich Ascites Carcinoma (EAC) cells:}

Ascetic fluid was withdrawn under aseptic conditions from tumor-bearing mice by needle aspiration from peritoneal cavity. Seven to ten days after EAC cells implantation, EAC cells were tested for viability and contamination using Trypan blue dye exclusion technique (Lazarus et al., 1966 ). Only EAC cells with at least $90 \%$ viability were used. EAC cells were suspended in normal saline so that each $0.1 \mathrm{ml}$ contains $2.5 \times 106$ cells. Cells were counted under the microscope using hemocytometer.

\section{Induction of solid tumors}

Each mouse was inoculated intradermally at 2 sites bilaterally on the lower ventral side (after shaving this area) with $100 \mu \mathrm{l}$ EAC suspension $(2.5 \times 106$ cells $)$ on each site.

\subsubsection{Study design:}

Animals were randomly divided into 7 groups, 10 animals each. Group (1) received the vehicle PBS (phosphate buffer saline) and was considered as normal group. Group (2) served as the EAC control untreated 
group. Group (3) received the Cisplatin as a standard drug (i.p.) at a dose of $2 \mathrm{mg} / \mathrm{kg}$ (Williams et al., 2001 ). Groups (4 \& 5) received benzoimidazoquinazoline (i.p.) at doses of $5 \mathrm{mg} / \mathrm{kg}$ and $10 \mathrm{mg} / \mathrm{kg}$ respectively. Groups ( $6 \&$ 7) received benzimidazotriazin (i.p.) at doses of $5 \mathrm{mg} / \mathrm{kg}$ and $10 \mathrm{mg} / \mathrm{kg}$ respectively. The synthetic compounds were dissolved in PBS as a vehicle. Cisplatin was available as vials ready to use. All treatments started at the $7^{\text {th }}$ day after inoculation EAC cells and were continued daily for 14 days except for cisplatin which was given every 3 days for two weeks.

\subsubsection{Sample Collection:}

At the $21^{\text {st }}$ day of the study, blood samples were collected from the orbital sinus (retro-orbital plexus), under light ether anesthesia, by a clean sterile capillary tube that was inserted at the inner canthus of the eye. The blood was left to be clotted for 20 minutes then centrifuged at $4000 \mathrm{rpm}$ for $15 \mathrm{~min}$. Serum samples were separated, collected and stored at $\left(-20^{\circ} \mathrm{C}\right)$ for determination of liver enzymes, urea, creatinine, and vascular endothelial growth factor (VEGF). RNA was also isolated from stored serum to assess the expression of miRNA 122. After cervical dislocation, tumor discs were separated, weighed and fixed in $10 \%$ neutral buffered formalin for immunohistochemical investigations.

1. Methods of analysis:

2. Determination of liver and kidney functions:

Serum activity of aspartate transaminase (AST) and alanine transaminase (ALT) enzymes were measured by enzymatic colorimetric method (Biodiagnostic, Egypt). Serum urea and creatinine levels were measured calorimetrically (Diamond, Egypt). The procedures were done according to the manufacturer's instructions.

\section{Determination of vascular endothelial growth factor (VEGF):}

Serum VEGF levels were determined using Quantikine@ ELISA kit purchased from R\&D systems (USA). The procedures were done according to the manufacturer's protocol, and the concentration was expressed as $\mathrm{pg} / \mathrm{g}$ tissue.

\section{Determination of miRNA-122 expression by quantitative real time polymerase chain reaction (qPCR):}

Total RNA, including small RNA, was isolated from serum samples using Qiagen miRNeasy Mini kit (Cat. No. 217004) (Qiagen, Germany) following the protocol supplied by the manufacturer. RNA concentration was determined by using NanoDrop spectrophotometer (Thermo Fisher Scientific Inc., USA).). MicroRNA122 expression was quantified by qRT-PCR. RNA was converted to cDNA by TaqMan(r) microRNA reverse transcriptase kit (Cat. No. 4366596) and Taqman assays hsa-miRNA-122 (5x) and RNU6B (5x) (Cat. No. 4427975) (Applied Biosystems, USA). MiRNA-122 expression was assessed against RNU6B as an endogenous control where $\Delta \mathrm{C}_{\mathrm{T}}$ and fold change were calculated. Assays used for amplification were has-miR- $122(20 \times)$ and RNU6B (20×) (Cat. No. 4427975) (Applied Biosystems, USA). The PCR reactions were performed in 20 $\mu \mathrm{L}$ total volume, including $10 \mu \mathrm{L}$ TaqMan@ Universal Master Mix (Cat. No. 4440043) (Applied Biosystems, USA), $1 \mu \mathrm{L}$ from each assay, $4 \mu \mathrm{L}$ complementary DNA (cDNA), and $5 \mu \mathrm{L}$ RNase- free water. PCR conditions consisted of $1 \mathrm{~min}$ at $95{ }^{\circ} \mathrm{C}$, followed by 40 cycles of $95^{\circ} \mathrm{C}$ for $15 \mathrm{~s}$ and $60{ }^{\circ} \mathrm{C}$ for $1 \mathrm{~min}$ and a final extension at $72{ }^{\circ} \mathrm{C}$ for 5 min. Real-time PCR was conducted using StepOnePlus Real-Time PCR thermal cycler (Applied Biosystems, USA).

\section{Immunohistochemistry}

Tumor discs for immunohistochemical examination were fixed in $10 \%$ neutral buffered formalin overnight and then embedded in paraffin. When analyzed, all paraffin-embedded tissues were sectioned at 4- $\mu \mathrm{m}$, deparaffinized in xylene, and hydrated through graded ethyl alcohol series in decreasing concentrations (100\%, $90 \%, 80 \%$ and $70 \%)$. Antigen retrieval was performed according to Tris/EDTA buffer $(\mathrm{pH}=9)$ antigen retrieval protocol. Tumor discs were stained applying the EnVision FLEX HRP labeled, High pH, method according to the manufacturer's staining protocol (Dako). Endogenous peroxidase activity was quenched by incubating the specimen for 10 min with phosphate buffer containing $3 \%$ hydrogen peroxide. Sections 
were incubated with corresponding primary antibodies at $4^{\circ} \mathrm{C}$ overnight. After conjugation with dextran coupled with peroxidase molecules and goat secondary antibody molecules against rabbit immunoglobulins, sections were incubated in DAB substrate chromogen for 2 minutes. Finally, Mayer's hematoxylin was used for counter staining.

\section{Evaluation of antibodies expression}

ImageJ was used for the semi-quantitative analysis of the immunohistochemical reactions. The images were captured by an optical microscope with a 40X objectives (Optika B-352A, Italia) coupled to a camera (HDCE30C) using its software and quantified using the ImageJ MacBiophotonics (National Institutes of Health, USA) software package developed by McMaster University (Ontario, Canada). The expression of VEGFR2 \& CD34 were assessed and percentage of stained area was measured

\section{Statistical analysis:}

All data were presented as mean \pm standard deviation (SD). Differences among groups was assessed using unpaired Student's t test and one-way ANOVA. P value less than 0.05 was considered to be statistically significant. Calculations were performed with the SPSS 21.0 statistical software package (IBM, USA).

\section{RESULTS}

\section{Effect on tumor weight:}

Tumor discs were removed and weighed on the 21st day of treatment. All treatment groups showed a significant decrease in the tumor weight compared to the EAC control group $(\mathrm{p}<0.01)$ except for the group treated with benzoimidazoquinazoline $(5 \mathrm{mg} / \mathrm{kg}$ ) where the average tumor weight was significantly higher than that observed in the standard cisplatin group $((\mathrm{p}<0.01)$ (Fig. 2$)$.

\section{Effect on liver and kidney functions:}

ALT and AST levels were increased in the EAC control mice and in all treatment, groups compared to the normal group (Table 1 ). However, the observed increase cannot be considered as an indication of liver toxicity. Creatinine and urea levels were also increased in the control mice compared to the normal group. Expectedly, creatinine and urea levels increased significantly in the cisplatin treated group compared to the control group $(\mathrm{p}<0.01)$. Treatment with the investigated compounds, benzoimidazoquinazoline $(\mathrm{C} 1)$ and benzimidazotriazine $(\mathrm{C} 2)$, in both doses $(5 \mathrm{mg} / \mathrm{kg}$ and $10 \mathrm{mg} / \mathrm{kg})$ showed significantly lower levels of creatinine and urea compared to the cisplatin treated group $(\mathrm{p}<0.05)$.

\section{Effect on serum levels of VEGF:}

VEGF serum level was evaluated in the current study as a marker for angiogenesis. VEGF levels were significantly higher in the EAC control group versus the normal mice $(\mathrm{p}<0.001)$. The serum levels of VEGF decreased significantly on treatment with cisplatin, the higher dose of benzoimidazoquinazoline, and both doses of benzimidazotriazine $(\mathrm{p}<0.05)$, while the lower dose of benzoimidazoquinazoline had no effect on VEGF levels (Fig. 3) .

\subsection{Effect on the expression of VEGFR2 and CD34:}

Immunohistochemical analysis showed that that EAC control group had high expression of VEGFR2, the main responder to VEGF signal that regulates endothelial migration and proliferation. Significant reduction in expression was observed in cisplatin treated group $(\mathrm{p}<0.001)$. Treatment with the test compounds in both doses decreased the expression compared to control EAC $(\mathrm{p}<0.001)$, but was still significantly higher than the cisplatin treated group (Fig. 4). The highest expression of CD34 was observed in the control EAC group. All other treated groups showed significantly lowered expression compared to control $(\mathrm{p}<0.001)$. Expression of CD34 in the group treated with the higher dose of benzimidazotriazine $(10 \mathrm{mg} / \mathrm{kg})$ was not significantly different from that of the cisplatin treated group

(Fig. 4). 


\subsection{Effect on levels of expression of miRNA-122 in tumor tissue:}

MiRNA-122 expression levels were determined in the tumor tissue of sacrificed mice. The expression of miRNA-122 was significantly downregulated in the mice with induced breast cancer $(\mathrm{p}<0.001)$ compared to the normal group. Its expression was significantly upregulated in all treatment groups. Treatment with Benzoimidazoquinazoline $(10 \mathrm{mg} / \mathrm{kg})$ gave almost similar expression levels as the group treated with cisplatin. The expression of miRNA-122 was significantly higher in both groups treated with benzimidazotriazine compared to the cisplatin group $(\mathrm{p}<0.05)$. Interestingly, the expression levels upon treatment with the higher dose of benzimidazotriazine $(10 \mathrm{mg} / \mathrm{kg})$ were not significantly different from the normal group (Fig. 5) .

\subsection{Molecular Modeling}

Computer-aided drug design simulation could save the effort, time, and resources required by traditional drug development methodology. Molecular modeling was performed to comprehend the potential interactions of the target compounds with VEGFR. Modulation activity of pazopanib and sorafenib derivatives towards VEGFR receptor was detected using glide docking of Schrodinger program. The docking study showed that the docking profile of the target compounds were at the pazopanib binding site (Fig. 6 ). Thys they are expected to have a similar mode of action. The binding of Pazopanib to VEGFR in its inactive conformation is believed to be similar to its interaction with its originally recognized kinase target B-RAF, a component of the RAS signal transduction network. Assuming so, the pyrimidine nitrogen in its un-ionized form and the nearby aniline NH moiety H-bond with the amide group of Cys919 in the hinge region ( $\mathrm{NH}$ and carbonyl oxygen, respectively). The benzimidazole moiety binds in a hydrophobic pocket, possibly occupying the site normally reserved for Phe1047 in the "DFG-in" (active) conformation. Compound 1 and Compound 2 were docked well in Pazopanib biding site and set up many hydrogen bonds, hydrophobic and electrostatic interaction (Fig. 6 ). CYS919, PHE1046, GLU917, THR916, GLU885, PHE918, LUE840, ILE1044, ILE888, ILE892, CYS1045 were the main amino acids involved in interactions. Generally, quinazoline and triazine scaffold occupy a deep pocket of ATP binding site and form hydrogen bond with the main amino acid involved in phosphorylation processes, CYS919. Additionally, the two compounds could form extra-hydrogen bonding with certain amino acids in hinge region such as GLU917, GLU885, and THR916. While hydroxyphenyl and dichloro-phenyl were occupied in the hydrophobic pocket in DFG-in active conformation forming hydrophobic and van der Wall interaction with PHE 1046, PHE918, LUE840, ILE1044, ILE888, ILE892, and CYS1045. Triazine containing compound had highest docking score of -9.1 and glide emodel of -62.6 , while compound with quinazoline moiety showed -8.5 and -87.4 of docking score and glide emodel comparing to that of pazopanib, -9.5, and -93.6.

\subsection{Lipinski's rule and in silico ADME prediction of the designed compounds}

The pharmacokinetic parameters of the proposed molecules were determined utilizing ADME prediction by Schrodinger QikProp v4.3. The proposed molecules were surveyed for their primary parameters of Lipinski's rule of 5 and other pharmacokinetic parameters. Table 2 showed the results got from QikProp with their reasonable qualities. Orally dynamic compounds should not violate the Lipinski rule multiple times. The designed compounds in the current study did not reach maximum permissible violations, indicating they have medication similarity properties. Oral bioavailability of medication-like molecules is constrained by the number of rotatable bonds (0-15) and polar surface area (7-200 $\AA$ ). The two compounds had a reasonable rotatable bond and polar surface area compared to pazopanib.

Intestinal absorption and permeation are important factors for drug absorption. They were assessed by the predicted Caco-2 cell permeability (QPPCaco), which is used as a model for the gut-blood barrier by nonactive transport. Caco-2 cell permeability of the proposed compounds were greater than $638 \%$ compared to pazopanib, indicating excellent drug permeability. The oral absorption of the designed compounds is superior to pazopanib. QPPMDCK descriptor was utilized to predict permeability of the proposed compounds through the blood brain barrier. MDCK cells are an excellent mimic of the blood-brain barrier. Both compounds showed excellent BBB permeability (QPPMDCK $>25)$ in comparison to pazopanib. This factor should be 
considered in future to diminish side effects. Human serum albumin binding capability was predicted with the QPlogkhsa descriptor of QikProp. The proposed compounds exhibited good binding capability to plasma proteins. The other physicochemical properties of the current two structures are listed in Table 2.

\section{DISCUSSION:}

Despite the improved survival rates of breast cancer patients, metastatic breast cancer is still considered a hazardous disease (Eroles et al., 2012). The current study aimed to investigate the effect of two novel heterocyclic compounds with a potential role in the treatment of breast cancer. The higher dose of benzoimidazoquinazoline $(\mathrm{C} 1)$ and both doses of benzimidazotriazine $(\mathrm{C} 2)$ showed a marked decrease in the tumor weight with a significant reduction of the angiogenic factor VGEF serum levels compared to the control group. All treatment doses significantly reduced the expression of VEGFR2 and CD34 in the tumor tissue. Our rational in this study is to design novel drug-like molecules that competitively bind to ATP binding pocket of VEGFR intracellular domain inhibiting its activity. The main feature to achieve that aim is to modulate the central core adenine ring of ATP or 2-aminopyrimidine of pazopanib, a strategy becomes common in medicinal chemistry to find novel potent VEGFR inhibitor with promising pharmacokinetic properties. A range of heterocyclic systems have been accounted to provide alternatives to adenine such as thienopyrimidines, quinoline, imidazopyridazine, and the pyrido pyrimidine (Fan-Wei et al., 2017, Holmes et al., 2007) . Herein, imidazoquinazoline and imidazotriazine were selected to mimic adenine and pyrimidine in hinge region presenting hydrogen bond to the key amino acid, cysteine (Fig 6 ). Moreover, the current ring system showed extra hydrogen bonds to certain amino acids in the hinge region giving the designed compounds tight binding to VEGFR. Pazopanib shows an amazing binding mode to VEGFR, where the indazole ring reaches out to the protein lipophilic pocket. In this study, we kept that feature by modifying indazole to hydroxyphenyl and dichlorophenyl, groups occupy the same pose of indazole forming van der Wall and hydrophobic interactions with key aromatic amino acids. Hydroxyl group of compound-2 shows extra hydrogen bonding with THR 916 in hydrophobic pocket giving this compound certain superiority. The proposed compounds, therefore, could be considered lead compounds which disrupt cell proliferation in solid tumor mediated by aberrant VEGF/VEGFR signaling pathway.

The VEGF and its receptor VEGFR2 play a major role in generating new blood vessels around the tumor mass. Binding of VEGF to its receptor induces endothelial cell proliferation and development. Inhibition of VEGFR2 and tumor angiogenesis are necessary for treatment of breast cancer (Cohen et al., 2001, Sebolt-Leopold et al., 2006 ). VEGF is associated with poor prognosis in breast cancer as there is increased synthesis in breast cancer tissues (Maae et al., 2006 ). VEGF can stimulate proliferation and migration of naturally inert endothelial cells during tumor angiogenesis and growth resulting in the formation of new vessel structures. Hypoxia is considered as the main cause of tumor angiogenesis, even though growth factors and a variety of transcriptional regulators are able to stimulate VEGF production through paracrine or autocrine mechanisms (Fox et al., 2007, Jia et al., 2013 )The higher dose of benzoimidazoquinazoline (C1) and both doses of benzimidazotriazine (C2) caused a marked decrease in the tumor weight and reduction in serum levels of the angiogenic factor VGEF. All treatment doses significantly reduced the expression of VEGFR2 and CD34 in the tumor tissue.

Assessment of tumoral micro-vessel density using immunohistochemical endothelial markers like factor CD31, CD34, and the activated endothelial cell marker CD105 is a commonly used procedure for measuring tumor angiogenesis in breast cancer (Uzzan et al., 2004 ). Benzimidazotriazine, compound C2-showed a promising activity in suppressing CD34 expression in mice bearing EAC. It has been proposed that tumor progression and metastasis in breast cancer is angiogenesis dependent (Pyakurel et al., 2014 ) The relation between the VEGF and CD34 expression has been reported in various malignant tumors. Thus, measuring their expression is vital for deciding the prognosis of tumor patients.

Reports showed that miRNA-122 is dysregulated in several cancer types, including liver, breast and lung cancers (Hopcraft et al., 2015, Ahsani et al., 2017, Akuta et al., 2016 ) In hepatocellular carcinoma (HCC), overexpression of miRNA-122 stimulates cell cycle arrest and apoptosis by inhibiting Cyclin G1 and Bcl-2 like 2 expression (Von Felden et al., 2017 ) On another hand, the overexpression of miRNA- 
122 in non- small cell lung cancers (NSCLC) may result in reducing the number of invasion and migration cells (These data confirm the hypothesis that miRNA-122 acts as a tumor suppressor in various types of cancer (Qin et al., 2015 ). The roles of specific miRNA in regulating tumor-associated angiogenesis and lymphangiogenesis have been reported., miRNA-20b is implicated in the regulation of VEGF in breast cancer cells by targeting HIF-1 verified the role of miRNA-10b as an angiogenic regulator (Cascio et al., 2010, Liu et al., 2017 ) miR-128 acts as a tumor suppressor through reducing the expression of both VEGFA and VEGFC (Hu et al., 2014 ).

miRNA-122 is involved in various cancers where they function as oncogene/tumor suppressor. Thus, it can serve as a diagnostic biomarker in cancer (Duc Duy et al., 2014 ). Perez-Anorve et al. reported that miRNA-122 is up-regulated in breast cancer cell. Functional analysis showed that miR-122 plays dual function as a tumor suppressor and oncomirR dependent on the tumor cells by diminishing endurance and advancing radiosensitivity (Isidro et al., 2019 ). Many studies have targeted miRNA-122 for disease management (Emile et al., 2020 ). Benzoisoquinoline derivatives activated miR-122 in hepatocarcinoma and function in the treatment of $\mathrm{HCV}$ infection by inducing apoptosis and reducing cell viability viacaspase activation. These compounds are considered promising lead molecules (Emile et al., 2020, Young et al., 2010 ) Expression of miRNA-122 in breast cancer tissue was significantly upregulated on treatment with both compounds, especially with benzimidazotriazine. Benzoisoquinoline was replaced by benzoimidazoquinazoline and benzimidazotriazin in an attempt to investigate the role of heterocyclic systems in mir-122 modulation. Both systems kept the modulation activity towards miR-122 activation. As a result, they may be a valuable tool for exploring the regulation of miR-122 in breast cancer and can possibly provide new targets and lead structures for the advancement of new chemotherapeutics.

A large number of drug-like molecules cure infectious diseases and treat cancer but have adverse effects on the host. In the current study, groups treated with benzoimidazoquinazoline and benzimidazotriazin had lower urea and creatinine levels compared to the cisplatin treated group, indicating a minimal nephrotoxic effect. Physicochemical properties also play an important role in deciding if a compound is suitable for therapeutic use. Solubility directly affects absorption; any therapeutic compound has to dissolve in biological fluids to effectively pass through a biological membrane. The designed compounds had the appropriate solubility as indicated by the partition coefficient (QplogPO/W). Orally active medications must be absorbed in the GIT and should penetrate the cellular membrane to reach their proposed targets. Benzoimidazoquinazoline and benzimidazotriazine had excellent permeability and oral absorptivity. Moreover, the novel molecules exhibited promising binding to plasma protein and can penetrate BBB, criteria essential in drug-like molecules. The physiochemical properties of the compounds determined in silico along with their anti-cancer effects in vivo in EAC animals give the compounds potential for use in treatment of breast cancer and warrant further clinical study.

\section{CONCLUSION}

Modulation of the activity of VEGF/VEGFR and mi-RNA-122 is a promising for the management of cancer. Two heterocyclic compounds, benzoimidazoquinazoline and benzimidazotriazine, exhibited anticancer properties through reducing the angiogenesis effect and enhancing the expression of miRNA-122. The efficacy of the two molecules at inhibition of VEGFR was validated in vitro, in vivo, and in silico using molecular modeling. The designed compounds anchored inside the hinge region of VEGFR forming a hydrogen bonding with key amino acids; cysteine mimics the adenine ring of ATP. Both compounds induced up-regulation of liver specific miRNA-122. The small molecules perturbed angiogenesis of EAC in mice by inhibition of both CD34 and VEGF/VEGFR signaling pathway. These data indicate that both molecules can be used as therapeutics for the treatment of breast cancer, with a better toxicity profile.

\section{ACKNOWLEDGMENT}

The authors are thankful to staff member of Pharmacology Department, Faculty of Pharmacy, Suez Canal University for help and support

\section{CONFLICT OF INTEREST}


The authors declare no competing financial interest

\section{REFRENCES}

Ahsani, Z., Mohammadi-Yeganeh, S., Kia, V., Karimkhanloo, H., Zarghami ,N., Paryan ,M.(2017) WNT1 gene from WNT signaling pathway is a direct target of miR-122 in hepatocellular carcinoma. Appl Biochem Biotechnol 181: 884- 897.

Akuta, N., Kawamura, Y., Suzuki, F., Saitoh ,S., Arase, Y., Kunimoto H., Sorin, Y., Fujiyama, S., Sezaki ,H., Hosaka, T., et al.(2016) Impact of circulating miR-122 for histological features and hepatocellular carcinoma of nonalcoholic fatty liver disease in Japan. Hepatol Int 10: 647-656.

Bray, F., Ferlay, J., Soerjomataram, I., Siegel, R.L., Torre, L.A., Jemal, A. (2018) Global cancer (statistics 2018: GLOBOCAN estimates of incidence and mortality worldwide for 36 cancers in 185 countries. CA Cancer J. Clin. 68, 394-424.

Cascio, S., D'Andrea, A., Ferla, R., Surmacz, E., Gulotta, E., Amodeo, V., Bazan, V., Gebbia, N., Russo, A.(2010) miR-20b Modulates VEGF Expression by Targeting HIF-1 Alpha and STAT3 in MCF-7 Breast Cancer Cells. J. Cell. Physiol. 224, 242-249.

Chen ,Z., Xu, S., Xu, W., Huang, J., Zhang ,GU., Lei, L., Shao, X., Wang, X. (2015) Expression of cluster of differentiation 34 and vascular endothelial growth factor in breast cancer, and their prognostic significance, Oncol Lett. 723-729

Cohen, P. (2001) Protein kinases-the major drug targets of the twenty-first century. Nat. Rev. Drug Discovery 1: 309-315.

Colozza, M., de Azambuja, E., Personeni, N., Lebrun, F., Piccart, M.J., Cardoso, F. (2007) Achievements in Systemic Therapies in the Pregenomic Era in Metastatic Breast Cancer. Onc. 12, 253-270.

Duc Duy ,Vo., Cathy, Staedel., Laura ,Zehnacker., Rachid, Benhida.,Fabien ,Darfeuille., and Maria, Duca.(2014) Targeting the Production of Oncogenic MicroRNAs with Multimodal Synthetic Small Molecules. ACS Chem. Biol. 9, 711-721

Emile ,N.,Van Meter Jackline ,A.,Onyango Kelly, A.Teske. (2020) A review of currently identified small molecule modulators of microRNA function. European Journal of Medicinal Chemistry, Volume 188, 112008

Eroles, P., Bosch, A., Perez-fidalgo, J.A., Lluch, A. (2012) Molecular biology in breast cancer: Intrinsic subtypes and signaling pathways. Cancer Treat. Rev. 38, 698-707.

Fan-Wei ,Peng.,Da-Ke, Liu.,Qing-Wen, Zhang.,Yun-Gen ,Xu \&Lei, Shi. (2012-2016) VEGFR-2 inhibitors and the therapeutic applications thereof: a patent review. Expert Opinion on Therapeutic Patents Volume 27,2017 - 987-1004

Ferrara, N., Gerber ,H.P., LeCouter, J. (2003) The biology of VEGF and its receptors. Nat Med. 9, 669-676.

Fox, S.B., Generali, D.G., Harris, A.L. (2007) Breast tumour angiogenesis. Breast Cancer Res 9: 216. doi: 10.1186/bcr1796 PMID: 18190723.

Hamam, R.,Hamam, D., Alsaleh, K.A., Kassem, M.,Zaher, W., Alfayez, M., Aldahmash, A., Alajez, N.M.(2017) Circulating microRNAs in breast cancer: Novel diagnostic and prognostic biomarkers. Cell Death Dis. 8, e3045.

Holmes, K., Roberts, O. L., Thomas, A. M. \& Cross, M. J. (2007) Vascular endothelial growth factor receptor-2: Structure, function, intracellular signalling and therapeutic inhibition. Cellular Signalling 19, 2003-2012.

Hopcraft ,S.E., Azarm, K.D., Israelow, B., Leveque, N., Schwarz, M.C., Hsu, T.H., Chambers, M.T., Sourisseau, M., Semler, B.L., Evans, M.J.(2015) Viral determinants of miR-122-independent hepatitis C virus replication. mSphere 1: 1. 
Howlader, N., Noone, A. M.,Krapcho, M., Neyman, N., Aminou, R., ltekruse, S. F.,Kosary, C. L., Ruhl, J., Tatalovich, Z., Cho, H., et al. (2009) SEER Cancer Statistics Review,1975-2009, Vintage Populations. Natl. Cancer Inst. 2012. Bethesda, MD.

Hu, F., Xu, P., Sun, B., Xiao, Z.(2017) Differences in the MicroRNA profiles of subcutaneous adipose derived stem cells and omental adipose -derived stem cells. Gene 625: 55 -63.

Hu, J., Cheng, Y., Li, Y., Jin, Z., Pan, Y., Liu, G., Fu, S., Zhang, Y., Feng, K., Feng, Y.(2014) microRNA-128 Plays a Critical Role in Human Non-Small Cell Lung Cancer Tumourigenesis, Angiogenesis and Lymphangiogenesis by Directly Targeting Vascular Endothelial Growth Factor-C. Eur. J. Cancer. 50, 2336-2350.

Isidro, X., Perez-Anorve, Claudia ,H., Gonzalez-De , Rosa., Ernesto Soto-Reyes., Fredy, O., BeltranAnaya, Oscar Del Moral-Hernandez, Marisol Salgado-Albarran, et al. (2019) New insights into radioresistance in breast cancer identify a dual function of miR-122 as a tumor suppressor and oncomiR. Mol Oncol. $13,1249-1267$.

Jia, D., Hasso, S.M., Chan, J., Filingeri ,D., D’Amore, P.A., Rice, L., et al.( 2013) Transcriptional repression of VEGF by ZNF24: mechanistic studies and vascular consequences in vivo. Blood 121: 707-715. doi: 10.1182/blood-2012-05-433045 PMID: 23212515.

Kian, R., Moradi, S.,Ghorbian, S. (2018) Role of components of microRNA machinery in carcinogenesis. Exp. Oncol. 40, 2-9.

Lala, P. K., Nandi, P., Majumder, M. (2018) Roles of Prostaglandins in Tumor Associated Lymphangiogenesis with Special Reference to Breast Cancer. Cancer Metastasis Rev. 37, 1.

Lazarus, H., Tegeler, W., Mazzone, H. M., Leroy, J. G., Boone, B. A., \& Foley, G. E.(1966) Determination of sensitivity of individual biopsy specimens to potential inhibitory agents: evaluation of some explant culture methods as assay systems. Cancer Chemotherapy Reports, 50(8), 543-555.

Liu, X.,Guan, Y., Wang, L.,Niu, Y.(2017) MicroRNA-10b Expression in Node-Negative Breast CancerCorrelation with Metastasis and Angiogenesis. Oncol. Lett. 14, 5845-5852.

Lou, W., Liu, J., Gao, Y.,Zhong, G., Chen, D., Shen, J., Bao, C., Xu, L., Pan, J., Cheng, J.,et al. (2017) MicroRNAs in Cancer Metastasis and Angiogenesis. Oncotarget 8, e115787.

Maae, E., Olsen, D.A., Steffensen, K.D., Jakobsen, E.H., Brandslund, I., Sorensen, F.B., et al. (2012). Prognostic impact of placenta growth factor and vascular endothelial growth factor A in patients with breast cancer. Breast Cancer Res Treat 133: 257-265. doi: 10.1007/s10549-012-1957-0 PMID: 22270936.

Maierthaler, M., Benner, A., Hoffmeister, M., Surowy, H., Jansen, L., Knebel, P., Chang-Claude, J., Brenner, H., Burwinkel, B.(2017) Plasma miR-122 and miR-200 family are prognostic markers in colorectal cancer. Int J Cancer 140: 176-187.

Musumeci, F., Radi, M., Brullo, C., et al. (2012) Vascular endothelial growth factor (VEGF) receptors: drugs and new inhibitors. J Med Chem. 55(24):10797-10822.

Olsson, A. K., Dimberg, A., Kreuger, J., Claesson-Welsh L. (2006) VEGF receptor signalling - in control of vascular function. Nat Rev Mol Cell Biol. 7:359-371

Otrock, Z.K., Mahfouz, R.A.R., Makarem, J.A., Shamseddine, A.I. (2007) Understanding the Biology of Angiogenesis: Review of the most Important Molecular Mechanisms. Blood Cells Mol. Dis. 39, 212-220.

Pan, C., Wang, X., Shi, K., Zheng, Y., Li, J., Chen, Y., Jin, L., Pan ,Z.(2016) miR-122 reverses the doxorubicin -resistance in hepatocellular carcinoma cells through regulating the tumor metabolism. PLoS One 11: e0152090.

Pyakurel, D., Karki, S., \& Agrawal, C. (2014) A study on microvascular density in breast carcinoma. Journal of Pathology of Nepal, 4(7), 570-575. 
Qin, H., Sha, J., Jiang, C., Gao, X., Qu ,L., Yan, H., Xu, T., Jiang, Q., Gao, H.(2015) miR-122 inhibits metastasis and epithelial-mesenchymal transition of non-small-cell lung cancer cells. Onco Targets Ther 8: 3175-3184.

Ran, S., Volk, L., Hall, K., Flister, M.J.(2010) Lymphangiogenesis and Lymphatic Metastasis in Breast Cancer. Pathophysiology 17, 229-251.

Sebolt-Leopold, J.S .(2006) Mechanisms of drug inhibition of signalling molecules. Nature 441: 457-462

Shibuya, M.(2011) Vascular Endothelial Growth Factor (VEGF) and its Receptor (VEGFR) Signaling in Angiogenesis: A Crucial Target for Anti- and Pro-Angiogenic Therapies. Genes Cancer 2, 1097-1105.

Song, S., Ajani, J.A.(2013) The role of microRNAs in cancers of the upper gastrointestinal tract. Nature Rev. Gastroenterol. Hepatol. 10, 109-118.

Svoronos, A.A., Engelman, D.M., Slack, F.J.(2016) Oncomir or tumor suppressor? The duplicity of microRNAs in cancer. Cancer Res. 76, 3666-3670.

Tao, L., Chu, L., Wang, L.I., Moy, L., Brammer, M., Song, C., Green, M., Kurian, A.W., Gomez, S., Clarke, C.A. (2016) Occurrence and outcome of de novo metastatic breast cancer by subtype in a large, diverse population. Cancer Causes Control. 27, 1127-1138.

Uzzan, B., Nicolas, P., Cucherat, M., Perret, G.Y. (2004) Microvessel density as a prognostic factor in women with breast cancer: a systematic review of the literature and meta-analysis. Cancer Res ;64:2941^55.

Von Felden, J., Heim ,D., Schulze, K., Krech, T., Ewald, F., Nashan, B., Lohse, AW., Wege, H.(2017) High expression of micro RNA-135A in hepatocellular carcinoma is associated with recurrence within 12 months after resection. BMC Cancer 17: 60, 2017

Wang, C., Zheng, X ., Shen, C., Shi, Y.(2012) MicroRNA-203 suppresses cell proliferation and migration by targeting BIRC5 and LASP1 in human triple-negative breast cancer cells. J Exp Clin Cancer Res. 31: 58.

Williams, J. I., Weitman, S., Gonzalez, C. M., Jundt, C. H., Marty, J., Stringer, S. D., Von Hoff, D. D.( 2001) Squalamine treatment of human tumors in $\mathrm{nu} / \mathrm{nu}$ mice enhances platinum-based chemotherapies. Clinical Cancer Research: An Official Journal of the American Association for Cancer Research, 7(3), 724-733.

Young, D.D., Connelly, C.M., Grohmann, C., Deiters, A. Small molecule modifiers of MicroRNA miR-122 function for the treatment of hepatitis C Virus infection and hepatocellular carcinoma.. Journal of the American Chemical Society 132, (23), 7976-7981

Zhong ,Hua., Qing ,Lv., Wenbin, Ye., Chung-Kwun Amy Wong, and Yaou ,Zhang. (2006) PLoS One. 2006; 1(1): e116.

Zorgetto ,V,A., Silveira ,G.G., Oliveira-Costa, J.P., Soave ,D.F., Soares, F.A., and Ribeiro-Silva, A.(2013) The relationship between lymphatic vascular density and vascular endothelial growth factor A (VEGF-A) expression with clinical-pathological features and survival in pancreatic adenocarcinomas. Diagn Pathol. 8:170.

Table 1: Liver enzymes and kidney markers in the study groups.

\begin{tabular}{|c|c|c|c|c|}
\hline & $\operatorname{ALT}(\mathrm{IU} / \mathrm{L})$ & $\overline{A S T}(\mathrm{IU} / \mathrm{L})$ & Urea $(\mathrm{mg} / \mathrm{dL})$ & Creatinine $(\mathrm{mg} / \mathrm{dL})$ \\
\hline Normal & $43.50 \pm 4.92$ & $52.87 \pm 5.13$ & $16.25 \pm 2.33$ & $0.91 \pm 0.07$ \\
\hline Control & $51.69 \pm 7.11^{*}$ & $62.16 \pm 7.99^{*}$ & $29.99 \pm 5.50^{*}$ & $1.82 \pm 0.19^{*}$ \\
\hline Cisplatin & $55.20 \pm 6.22^{*}$ & $67.34 \pm 8.25^{*}$ & $44.15 \pm 6.42^{*} \#$ & $4.88 \pm 1.11^{* \#}$ \\
\hline \multicolumn{2}{|c|}{$\begin{array}{l}\text { Benzoimidazoquinazolinøe. } 32 \pm 5.20^{*} \\
(5 \mathrm{mg} / \mathrm{kg})\end{array}$} & $61.13 \pm 6.30^{*}$ & $30.23 \pm 5.55^{* \$}$ & $1.77 \pm 0.22^{* \$}$ \\
\hline \multicolumn{2}{|c|}{$\begin{array}{l}\text { Benzoimidazoquinazoli.5e } 2.13 \pm 6.19^{*} \\
(10 \mathrm{mg} / \mathrm{kg})\end{array}$} & $63.50 \pm 6.80^{*}$ & $34.67 \pm 5.78^{* \$}$ & $2.01 \pm 0.28^{* \$}$ \\
\hline
\end{tabular}




\begin{tabular}{lllll}
\hline & ALT $(\mathrm{IU} / \mathrm{L})$ & AST $(\mathrm{IU} / \mathrm{L})$ & Urea $(\mathrm{mg} / \mathrm{dL})$ & Creatinine $(\mathrm{mg} / \mathrm{dL})$ \\
\hline $\begin{array}{l}\text { Benzimidazotriazine } \\
(5 \mathrm{mg} / \mathrm{kg})\end{array}$ & $53.15 \pm 6.20^{*}$ & $65.33 \pm 6.98^{*}$ & $35.38 \pm 5.60^{* \$}$ & $2.10 \pm 0.29^{* \$}$ \\
$\begin{array}{l}\text { Benzimidazotriazine } \\
(10 \mathrm{mg} / \mathrm{kg})\end{array}$ & $56.23 \pm 7.09^{*}$ & $68.23 \pm 8.11^{*}$ & $37.16 \pm 6.00^{* \$}$ & $2.55 \pm 0.78^{* \$}$ \\
\hline
\end{tabular}

Values are expressed as mean \pm SD. Data were analyzed using ANOVA followed by Bonferroni post hoc test. * significantly different compared to the normal group at $\mathrm{p}<0.05,{ }^{*}$ significantly different compared to the control group at $\mathrm{p}<0.05{ }^{\$}{ }^{\$}$ significantly different compared to the cisplatin group at $\mathrm{p}<0.05$.

Table 2: Results of ADME study for the designed compounds in comparison with Pazopanib.

\section{Comp.}

No.

Mol-MWa

DonorHB $^{b}$

AcceptHB $^{\text {c }}$

$\mathrm{QP} \log \mathrm{Po} / \mathrm{w}^{\mathrm{d}}$

\#rotor

PSA $^{\mathbf{f}}$

$\mathrm{QP}^{\mathrm{P} \log \mathrm{S}^{\mathrm{g}}}$

QPlogHERG $^{\mathrm{h}}$

QPPCaco ${ }^{\mathrm{i}}$

QPP

MDCK $^{j}$

QPlogKhsa $^{\mathrm{k}}$

\% Human

Oral

Absorption ${ }^{1}$

\section{Compound-1}

453.3

1

5.5

5.5

5

63.4 
$-7.6$

$-7.1$

2325

10000

0.714

100

Compound-2

276.2

2

4.7

2.4

3

62.8

$-4.1$

$-5.9$

916.1

450

$-0.03$

94.11

Pazopanib

437.5

3

8.5

2.7

6

115.1

$-5.88$

$-6.2$

124.7

53.3

0.265

80.6 
Figure 1. Chemical structures of US FDA approved drugs (VEGFR inhibitors) for cancer treatment and the designed compounds

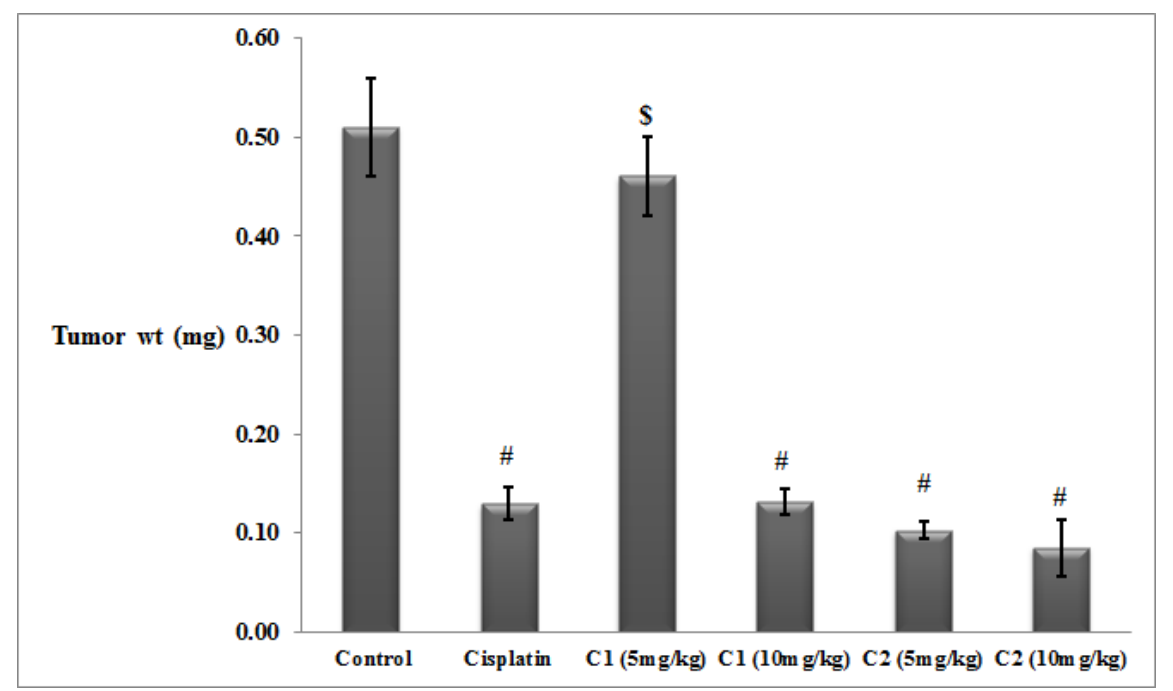

Figure 2: Effect of treatment on the tumor weight in EAC bearing mice. $\mathrm{C} 1=$ benzoimidazoquinazoline, $\mathrm{C} 2=$ benzimidazotriazine. Values are expressed as mean $\pm \mathrm{SD}$. All data were analyzed using ANOVA followed by bonferroni post hoc test. ${ }^{*}$ significantly different compared to the control group, ${ }^{\$}$ significantly different compared to the cisplatin group. Differences were considered significant at $\mathrm{p}<0.05$

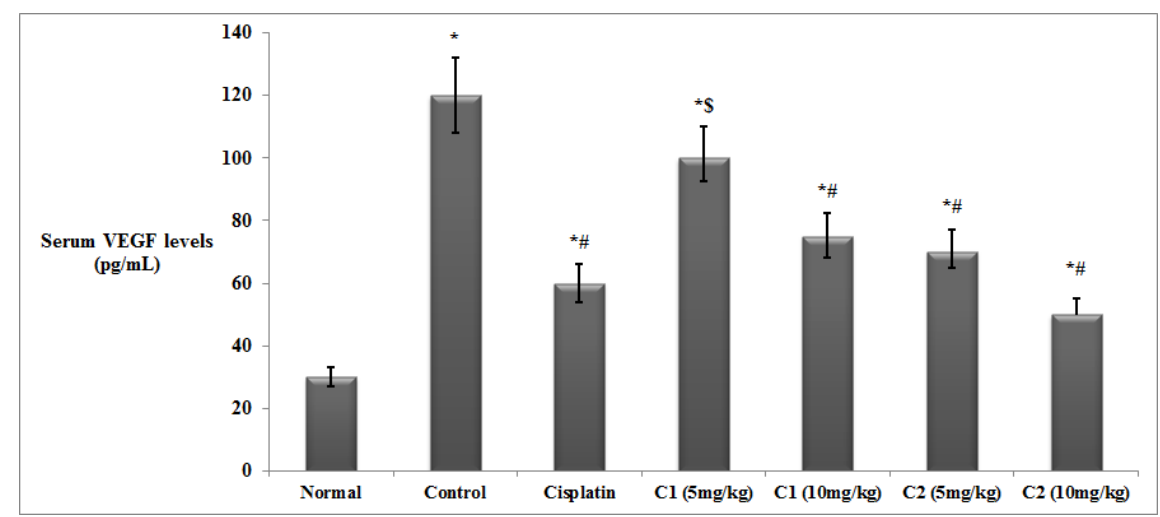

Figure 3: Levels of VEGF determined in the serum by ELISA. C1= Benzoimidazoquinazoline, $\mathrm{C} 2=$ Benzimidazotriazine. Values are expressed as mean \pm SD. All data were analyzed using ANOVA followed by bonferroni post hoc test. ${ }^{*}$ significantly different compared to the normal group, ${ }^{*}$ significantly different compared to the control group, ${ }^{\$}$ significantly different compared to the cisplatin group. Differences were considered significant at $\mathrm{p}<0.05$. 
(A) VEGFR2

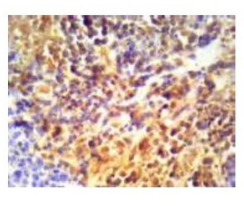

Control

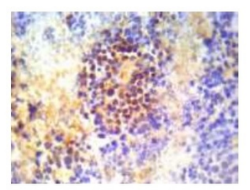

$\mathrm{Cl}(5 \mathrm{mg} / \mathrm{kg})$

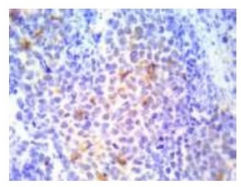

$\mathrm{C} 2(5 \mathrm{mg} / \mathrm{kg})$

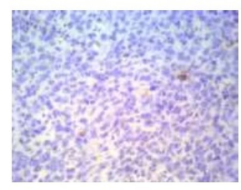

Cisplatin

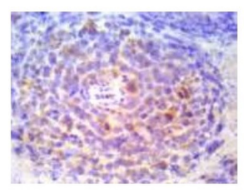

$\mathrm{C} 1(10 \mathrm{mg} / \mathrm{kg})$

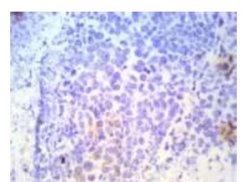

C2 $(10 \mathrm{mg} / \mathrm{kg})$
(B) $\mathrm{CD} 34$

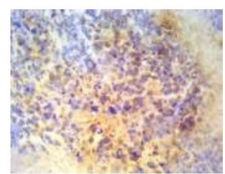

Control

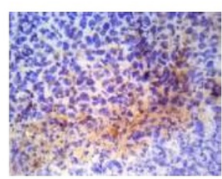

$\mathrm{C} 1(5 \mathrm{mg} / \mathrm{kg})$

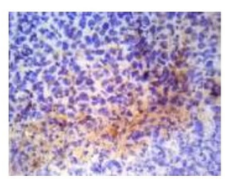

$\mathrm{C} 2(5 \mathrm{mg} / \mathrm{kg})$

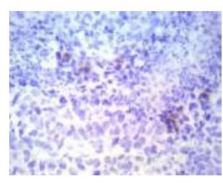

Cisplatin

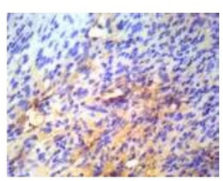

$\mathrm{Cl}(10 \mathrm{mg} / \mathrm{kg})$

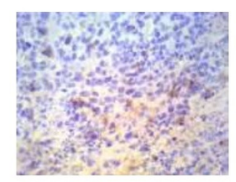

$\mathrm{C} 2(10 \mathrm{mg} / \mathrm{kg})$
C

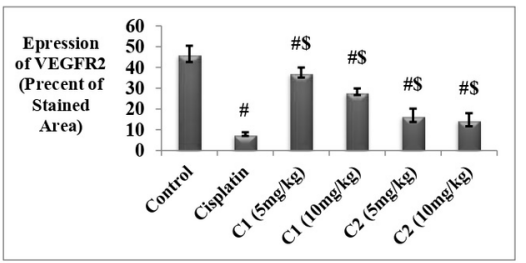

D

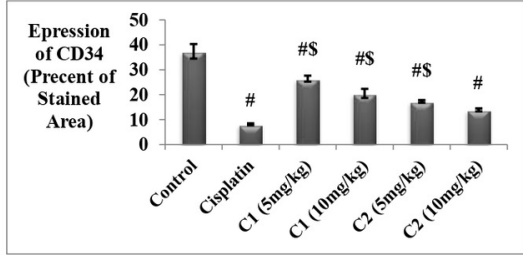

Figure 4: Expression of VEGFR2 and CD34 in tumor tissue.(A \& B) Representative photomicrographs of (A) VEGFR2 and (B) CD34. (C \& D) The percentage of positive immunohistochemical reactions (brown stained area) analysed by Image $\mathrm{J}$ software. $\mathrm{C} 1=$ benzoimidazoquinazoline, $\mathrm{C} 2=$ Benzimidazotriazine. Values are expressed as mean $\pm \mathrm{SD}$. The. Data were analyzed using ANOVA followed by Bonferroni post hoc test. \# significantly different compared to the control group, ${ }^{\$}$ significantly different compared to the cisplatin group. Differences were considered significant at $\mathrm{p}<0.05$.

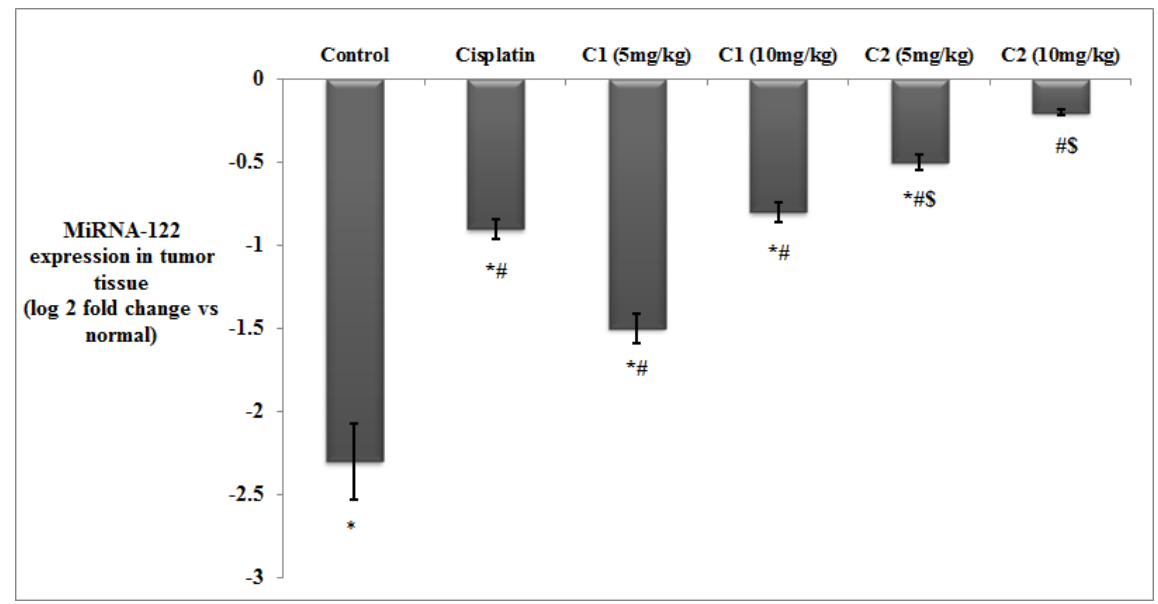


Figure 5: Levels of expression of miRNA-122 in tumor tissue determined by real time PCR. C1= benzoimidazoquinazoline, $\mathrm{C} 2=$ Benzimidazotriazine. Values are expressed as mean of $\log 2$ fold change vs the normal group $\pm \mathrm{SD}$. The expression of the normal group is normalized to 1 where $\log 2(1)=$ zero. Data were analyzed using ANOVA followed by Bonferroni post hoc test. ${ }^{*}$ significantly different compared to the normal group, $\#$ significantly different compared to the control group, ${ }^{\$}$ significantly different compared to the cisplatin group. Differences were considered significant at $\mathrm{p}<0.05$.

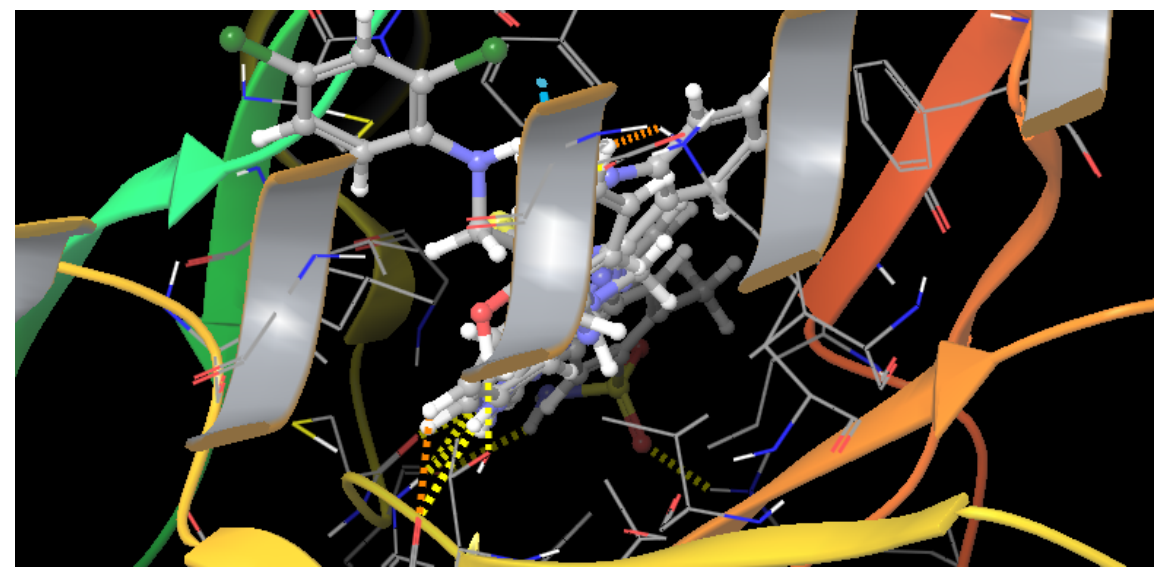

Figure 6 . (A) 3D binding mode and overlay of Pazopanib and the designed compounds in kinase domain of human VEGFR-2 (PDB 3EWH). 2D of ligand receptor interaction of (B) Benzimidazotriazine, (C) Benzoimidazoquinazoline, and (D) Pazopanib 